\title{
Grupo de Usabilidade e Engenharia de Software - USES
}

\author{
Tayana Conte, Adriana Lopes, Felipe Manzoni, Leonardo Marques, Márcia Lima, Patrícia Fernandes, \\ Ana Carolina Oran, Bruno Gadelha \\ Instituto de Computação (IComp) \\ Universidade Federal do Amazonas (UFAM) \\ Manaus/Brasil \\ \{tayana, adriana, fsm2, lcm, marcia.lima, patriciagfm, ana.oran, bruno\}@icomp.ufam.edu.br
}

\section{RESUMO}

Este artigo apresenta o grupo de pesquisa de Usabilidade e Engenharia de Software (USES) da Universidade Federal do Amazonas. As pesquisas do grupo USES visam desenvolver soluções nas áreas de Interação Humano-Computador (IHC) e de Engenharia de Software (ES) que beneficiem a academia e a indústria de software. Dentre as atividades de pesquisa do grupo destaca-se a caracterização de soluções para: (i) desenvolver sistemas interativos com foco na qualidade do processo de desenvolvimento e uso destes sistemas, (ii) apoiar o ensino de modelos de software e (iii) apoiar as atividades gerenciais durante o desenvolvimento de tais sistemas.

\section{PALAVRAS-CHAVE}

Interação Humano-Computador; Engenharia de Software

\section{INTRODUÇÃO}

O grupo de pesquisa de USabilidade e Engenharia de Software (USES) é vinculado ao Programa de Pós-Graduação em Informática (PPGI) da Universidade Federal do Amazonas (UFAM). O USES tem como objetivo desenvolver soluções nas áreas de Interação Humano-Computador (IHC) e Engenharia de Software (ES) através de pesquisas científicas que visem beneficiar tanto a academia quanto a indústria de software.

Neste contexto, na área de IHC, o grupo USES atua nos seguintes temas: Usabilidade, User eXperience (UX), Playability, Player Experience (PX), Design Thinking (DT) e Engenharia Semiótica. $\mathrm{Na}$ área de ES, o grupo USES atua nos seguintes temas: Engenharia de Software Experimental, Processo de Software, Qualidade de Software, Ensino de diagramas da UML (Unified Modeling Language), Engenharia de Requisitos, Gerência de Conhecimento, Fatores Humanos em ES e Developer eXperience (DX).

\footnotetext{
Permission to reproduce or distribute, in whole or in part, material extracted from this work, verbatim, adapted or remixed, as well as the creation or production from the content of such work, is granted without fee for non-commercial use, provided that the original work is properly credited.

IHC 2019 - TRILHA FÓRUM DOS GRUPOS DE PESQUISA, Outubro 21-25, 2019, Vitória, Brasil. In Anais Estendidos do XVIII Simpósio Brasileiro sobre Fatores Humanos em Sistemas Computacionais. Porto Alegre: SBC.

(C) 2019 by the author(s), in accordance with the terms of the Creative Commons Attribution-NonCommercial 4.0 International Public License (CC BY-NC 4.0).
}

\section{PESQUISADORES ENVOLVIDOS}

Os pesquisadores do grupo $\mathrm{USES}^{1}$ almejam promover a integração das atividades e responsabilidades das áreas de IHC e ES no desenvolvimento de sistemas interativos. A Figura 1 apresenta os pesquisadores do grupo USES. O grupo é composto por líderes, com doutoramento nas áreas de ES e IHC, por colaboradores, que atuam na indústria e academia, e por alunos de mestrado e doutorado. Alguns alunos possuem colaborações com outros pesquisadores.

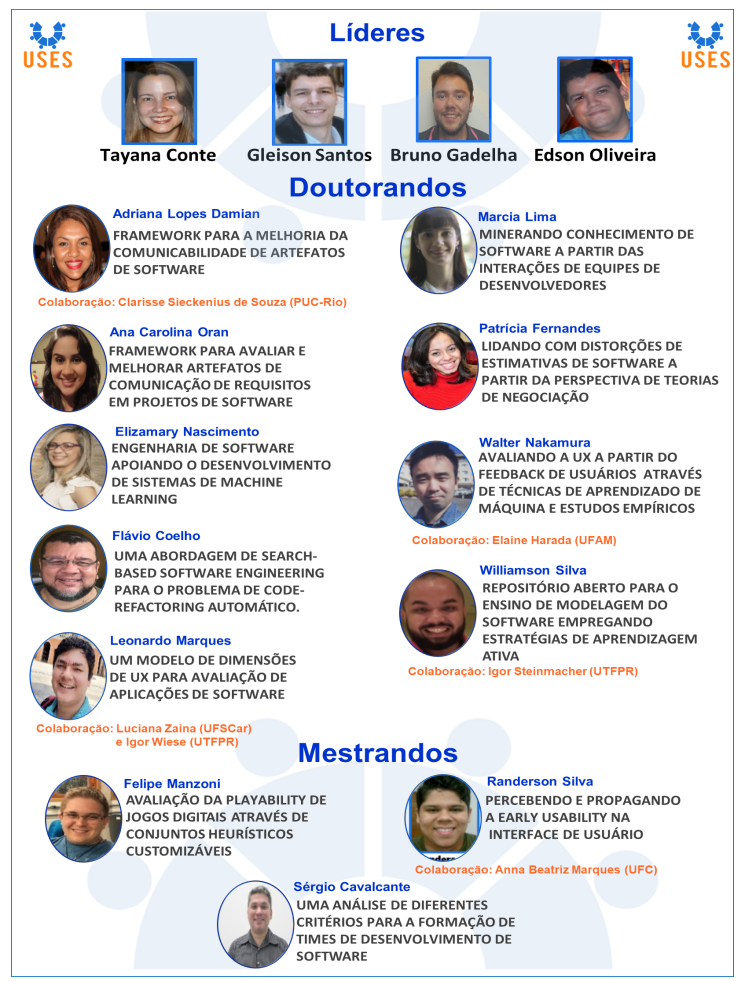

Figura 1: Grupo de Pesquisa USES em 2019.

\section{PRINCIPAIS PROJETOS DE PESQUISA}

Dentre os projetos de pesquisa vigentes e/ou concluídos do grupo USES, é possível destacar:

\footnotetext{
${ }^{1}$ Página do Grupo USES em http://uses.icomp.ufam.edu.br/
} 


\section{T. Conte et al.}

\subsection{Melhorando a Qualidade no Desenvolvimento de Software No Estado do Amazonas por Meio da Integração de Usabilidade no Processo de Desenvolvimento}

Projeto é financiado pela Fundação de Amparo à Pesquisa do Estado do Amazonas (FAPEAM), com o intuito de caracterizar novas tecnologias para o controle e a estimativa da usabilidade de sistemas interativos durante o processo de software, com ênfase nas etapas iniciais do desenvolvimento. As novas tecnologias possuem dois grandes focos: (i) na elicitação de requisitos de software, através do desenvolvimento de artefatos que apoiem a especificação e estimativa da qualidade dos requisitos de usabilidade; e (ii) em abordagens que auxiliem a projetar a usabilidade durante a criação de modelos de projeto de software.

\subsection{Apoiando o Design de Sistemas Interativos com Foco na Qualidade de Uso}

Projeto é financiado pelo Conselho Nacional de Desenvolvimento Científico e Tecnológico (CNPq), que visa realizar pesquisas científicas na interseção entre as áreas de IHC e ES. O intuito é apoiar o desenvolvimento de sistemas interativos com foco na qualidade de uso, através da utilização de modelos adequados. Dentre as contribuições esperadas, é possível destacar: (i) avaliação de modelos existentes em relação à sua adequação ao design de sistemas interativos; (ii) melhor compreensão sobre as dificuldades no ensino e aprendizagem de modelos para o design de sistemas interativos; (iii) apoio ao design de sistemas interativos com foco na qualidade de uso; e (iv) transferência de tecnologias desenvolvidas para a indústria de software através da realização de estudos experimentais.

\subsection{Cooperação Acadêmica em Sistemas de Recomendação para Manutenção Colaborativa de Software}

Este projeto conta com a colaboração de programas de pósgraduação da área de computação das universidades: Pontifícia Universidade Católica do Rio de Janeiro (PUC-Rio), Universidade Federal de Alagoas (UFAL), Universidade Federal de Campina Grande (UFCG) e UFAM. O projeto é financiado pela Coordenação de Aperfeiçoamento de Pessoal de Nível Superior (CAPES), que visa investigar como sistemas de recomendação podem apoiar efetivamente as tarefas de manutenção de software. Dentre as contribuições técnicas esperadas, é possível destacar: (i) sistemas de recomendação para auxiliar a manutenção colaborativa de sistemas de software e (ii) métodos que apoiem a avaliação da qualidade de uso de sistemas de recomendação.

\section{INFRAESTRUTURA PARA A EXECUÇÃO DAS PESQUISAS}

O grupo USES possui um laboratório de pesquisa que dispõe de uma infraestrutura adequada para que os pesquisadores possam realizar as suas atividades de pesquisa. O laboratório disponibiliza equipamentos de hardware e software compatíveis com as necessidades de realização de pesquisas do grupo, sendo todos adquiridos através de recursos provenientes de projetos de pesquisa (vigentes ou concluídos).

\section{SOLUÇÕES DESENVOLVIDAS}

Esta seção apresenta, resumidamente, as soluções desenvolvidas pelo grupo USES. Tais soluções estão organizadas pelos principais tópicos de pesquisa investigados pelo grupo USES.

\subsection{Desenvolvimento de Sistemas com Foco na Qualidade do Processo}

Diretivas de Comunicabilidade: diretivas que tem por objetivo melhorar a comunicação entre produtores e consumidores de artefatos de software, auxiliando estes produtores a refletirem sobre sua intenção de comunicação.

ReComP (Framework of Requirements Communication based on perspective): tecnologias que auxiliam a avaliação e a melhoria da comunicação de requisitos em projetos de software, baseada na perspectiva de desenvolvedores e testadores.

E-VOL BPMN (Evolution of BPMN): técnica que tem por objetivo auxiliar profissionais com pouco conhecimento na notação BPMN, na evolução dos modelos BPMN.

Soluções de data-mining para minerar repositórios de ES: métodos que identificam e extraem conhecimentos registrados em arquivos de logs produzidos a partir da interação das equipes de desenvolvimento com o apoio de algoritmos da área de Recuperação de Informação e Machine Learning (ML). Tais conhecimentos constituem informação relevante para apoiar a evolução e a qualidade de produtos de software desenvolvido.

CheckBM (Checklist to support Business Modeling) e CheckDP (Checklist to support in Data Processing): artefatos que apoiam desenvolvedores em tarefas essenciais no processo de desenvolvimento de sistemas de ML.

Atualmente, o grupo está: (i) desenvolvendo tecnologias baseadas em ML para apoiar a colaboração de equipes durante o desenvolvimento de software; (ii) investigando as práticas adotadas no desenvolvimento de sistemas de ML para apoiar outras equipes no desenvolvimento deste tipo de sistema; (iii) desenvolvendo diferentes soluções para melhorar a comunicação de equipes de desenvolvimento de software com base em estudos experimentais e teorias que investigam diferentes formas de comunicação.

\subsection{Desenvolvimento de Sistemas Interativos com Foco na Qualidade de Uso \\ Conjunto de técnicas MIT (Model Inspection Technique for Usability Evaluation): técnicas que orientam o inspetor durante a avaliação de usabilidade em especificações de Caso de Uso (MIT 1), em mockups (MIT 2) e em Diagrama de Atividades (MIT 3). UDG (Usability Design Guidelines): diretrizes para criação de mockups visando a usabilidade das aplicações. \\ REX (Redesigning for Experience): técnica que tem por objetivo auxiliar profissionais no processo de avaliação de UX e redesign de aplicações móveis.}


UDRT-AD (Usability Design Reading Technique for Activity Diagrams): técnica para projetar diagrama de atividades visando a melhoria da usabilidade em aplicações interativas.

UX-Tips (User eXperience Technique for Interactive ProductS): técnica de avaliação de UX com o diferencial de identificar problemas que possam ter causado uma UX negativa.

Técnicas MoLVERIC: técnicas que auxiliam na detecção e categorização de defeitos em diagramas MoLIC por meio de listas de verificação (MCheck) e cartas (MCards).

TUXEL (Technique for User eXperience Evaluation in Learning): técnica que apoia a realização da avaliação de usabilidade, usabilidade pedagógica e a UX em Ambientes Virtuais de Aprendizagem, de forma a possibilitar a melhoria do processo de ensino e aprendizagem.

USINN (USability-oriented INteraction and Navigation model): notação proposta para modelagem de interação e navegação considerando aspectos funcionais de usabilidade.

DTA4RE: assistente de apoio ao DT para elicitação de requisitos. Atualmente o grupo está desenvolvendo: (i) um modelo de dimensões de UX que contemple características importantes de serem consideradas ao avaliar um software; (ii) técnicas para a avaliação da Playability em jogos digitais, através do uso de heurísticas, para apoiar profissionais não especialistas; (iii) técnica para a avaliação da Player Experience em jogos de realidade virtual; (iv) pesquisas para a compreensão da Early Usability a partir dos artefatos que são utilizados para compreender o sistema interativo que será desenvolvido.

\subsection{Ensino de Modelos para o Desenvolvimento de Sistemas Interativos}

Modelo de dificuldades no aprendizado de casos de uso: o modelo representa as dificuldades percebidas por estudantes que estão aprendendo a modelar casos de uso, incluindo as dificuldades do diagrama e da descrição textual de casos de uso.

OpenSMALS: um repositório aberto que visa abordar os desafios práticos enfrentados pelos instrutores durante a aplicação de estratégias de aprendizado ativas.

Atualmente o grupo USES está conduzindo pesquisas para: (i) compreender o uso do OpenSMALS por diferentes instrutores; (ii) entender a influência das abordagens utilizadas por instrutores no ensino da UML, avaliando a percepção de estudantes como futuros profissionais da indústria de software.

\subsection{Atividades Gerenciais no Desenvolvimento De Sistemas Interativos}

Atualmente, o grupo está: (i) investigando como teorias de negociação podem ser usadas para evitar distorções em estimativas de projetos de software, de maneira a permitir que as equipes de desenvolvimento sejam capazes de defender suas estimativas quando são comunicadas para a alta gerência, durante o estabelecimento de compromissos de projetos e a criação de planos; e (ii) analisando critérios de formação de equipes de desenvolvimento de software na indústria, com o intuito de melhorar seu desempenho. $\mathrm{O}$ foco destas pesquisas consiste na melhoria da DX, a qual se refere às experiências do desenvolvedor no contexto do desenvolvimento de software, incluindo as suas atividades e os softwares que utiliza, relacionadas com aspectos cognitivos e emocionais.

\section{PARCERIAS COM OUTROS GRUPOS DE PESQUISA}

O grupo USES possui parcerias com outros grupos de pesquisa que atuam em IHC e ES. No contexto de pesquisas em IHC, o grupo SERG (Semiotic Engineering Research Group), da PUCRio, vem desenvolvendo junto ao USES soluções para o design de sistemas com foco na qualidade de processo e uso. O grupo USES tem colaborado com a professora Natasha Valentim do grupo Design de Interação para a Inclusão e o Desenvolvimento Social da UFPR no desenvolvimento de soluções com foco no usuário. Além disso, o USES possui parcerias com os grupos UXLeris da UFSCar e Hu-S.ER da PUCRS. No âmbito de ES, os grupos de pesquisa OPUS da PUC-Rio, ESE (Grupo de Engenharia de Software Experimental) da COPPE/UFRJ e SICCS (Software Engineering Research Laboratory) da Northern Arizona University têm colaborado em pesquisas focadas no apoio ao ensino de modelos e na qualidade do processo de desenvolvimento. Em ambas as áreas de IHC e ES, o grupo USES tem colaborado com os professores David Redmiles e Iftekhar Ahmed do grupo CRADL (Collaboration Research in Action, Design, and Learning) da University of California/Irvine e com a professora Anna Beatriz Marques do LINCE (Laboratório Interdisciplinar de Ciência da Computação e Engenharia de Software) da UFC/Campus Russas. Os grupos de pesquisa vêm realizando o intercâmbio de alunos do USES nas universidades em que estes atuam. Outras parcerias podem ser vistas na página do USES ${ }^{1}$.

\section{INTERESSES DE PARCERIAS DO GRUPO USES COM OUTROS GRUPOS DE PESQUISA}

O grupo de pesquisa USES tem a intenção de firmar outras parcerias com grupos de pesquisa das áreas de IHC e ES, envolvendo pesquisas relacionadas às soluções que estão sendo desenvolvidas. Além disso, o grupo de pesquisa pretende compreender melhor os tópicos de pesquisa relacionados à qualidade de processo e uso de sistemas, como soluções propostas para uma melhor DX e avaliação de usabilidade e UX de diferentes produtos de software, além do apoio às atividades gerenciais dos sistemas, como estimativas de projeto e alocação de membros de equipes de software. Além disso, o grupo pretende explorar outros tópicos que possam apoiar o desenvolvimento de soluções relacionadas às áreas de IHC e ES, como uso de Gamification aplicada à DX. A descrição dos tópicos de pesquisa apresentados são apenas indicações de interesse em parcerias, não se limitando a tais tópicos. Desta forma, o grupo USES espera desenvolver novas pesquisas nas áreas de IHC e ES.

\section{AGRADECIMENTOS}

Os autores agradecem o apoio financeiro do $\mathrm{CNPq}$ nos processos 423149/2016-4, 311494/2017-0 e 204081/2018-1/PDE; e CAPES através do processo 175956/2013. 